\title{
Motor neuron and pancreas homeobox 1/HLXB9 promotes sustained proliferation in bladder cancer by upregulating CCNE $1 / 2$
}

Mingkun Chen ${ }^{1 \dagger}$, Rongpei $\mathrm{Wu}^{2 \dagger}$, Gang Liं ${ }^{3+}$, Cundong Liu', Lei $\operatorname{Tan}^{4}$, Kanghua Xiao ${ }^{4}$, Yunlin Ye ${ }^{4,5^{*}}$ and Zike Qin ${ }^{4,5^{*}}$

\begin{abstract}
Background: Uncontrolled proliferation is thought to be the most fundamental characteristic of cancer. Detailed knowledge of cancer cell proliferation mechanisms would not only benefit understanding of cancer progression, but may also provide new clues for developing novel therapeutic strategies.

Methods: In vitro function of MNX1 (Motor neuron and pancreas homeobox 1) in bladder cancer cell was evaluated using MTT assay, colony formation assay, and bromodeoxyuridine incorporation assay. Real-time PCR and western blotting were performed to detect MNX1 and CCNE1/2 expressions. In vivo tumor growth was conducted in BALB/c-nu mice.

Results: We reported that MNX1 is responsible for sustaining bladder cancer cell proliferation. Abnormal MNX1 upregulation in bladder cancer cell lines and 167 human tissue specimens; high MNX1 expression levels correlated significantly with shorter 5-year overall and relapse-free survival in the bladder cancer patients. Furthermore, MNX1 overexpression accelerated bladder cancer cell proliferation and tumorigenicity both in vitro and in vivo, whereas MNX1 downregulation arrested it. In addition, MNX1 transcriptionally upregulated CCNE1 and CCNE2 by directly bounding to their promoters, which promoted G1-S transition in the bladder cancer cells.

Conclusion: These findings reveal an oncogenic role and novel regulatory mechanism of MNX1 in bladder cancer progression and suggest that MNX1 is a potential prognostic biomarker and therapeutic target.
\end{abstract}

Keywords: Bladder cancer, Proliferation, MNX1, CCNE1, CCNE2

\section{Background}

Bladder cancer is a primary cause of morbidity and mortality, with 429,700 estimated new cases each year and 165,000 deaths worldwide [1]. Treatment for bladder cancer has improved greatly in recent decades, however, many patients especially those at the advanced stages of the disease, succumb to it. Although localized bladder cancer can be excised by surgery, the recurrence and progression rates remain high. Patients with advanced bladder cancer mostly receive radiotherapy or chemotherapy, but the therapeutic

\footnotetext{
*Correspondence: yeyunlin2000@163.com; 351844459@qq.com

${ }^{\dagger}$ Mingkun Chen, Rongpei Wu and Gang Li contributed equally to this work.

${ }^{4}$ Department of Urology, Sun Yat-sen University Cancer Center, State Key

Laboratory of Oncology in South China, Collaborative Innovation Center for

Cancer Medicine, Guangzhou 510060, Guangdong, China

Full list of author information is available at the end of the article
}

outcomes remain unsatisfactory [2]. Therefore, there is an urgent need to determine the underlying molecular mechanisms of bladder cancer tumorigenesis and to develop effective, molecular targeted bladder cancer therapies.

The vital feature of cancer is thought to be sustained proliferation [3]. Generally, dysregulation in cell cycle progression results in the release of proliferative signals in cancer cells, consequently disrupting cell number homeostasis and causing uncontrolled cell proliferation. The prevailing model during cell cycle progression is that cyclin-dependent kinase (CDK) activity increases during G1-S transition, which involves the formation of a cyclin-CDK complex. Subsequently, phosphorylation of the retinoblastoma $(\mathrm{Rb})$ family members leads to the release of E2F-containing repressor complexes from E2F-regulated

(C) The Author(s). 2018 Open Access This article is distributed under the terms of the Creative Commons Attribution 4.0 International License (http://creativecommons.org/licenses/by/4.0/), which permits unrestricted use, distribution, and 
promoters and upregulates the expression of E2F downstream target genes [4]. In detail, CCNE (cyclin E, including CCNE1 and CCNE2) binds to CDK2, and subsequently phosphorylates $\mathrm{Rb}$ to promote $\mathrm{G} 1-\mathrm{S}$ progression. It is well-demonstrated that the dysregulation of CCNE-CDK2 activity is involved in many human cancers, including breast, bladder, and lung cancer, resulting in uncontrolled cell proliferation [5-8]. Consistently, CCNE overexpression is associated with poor clinical prognosis of bladder cancer, while inhibiting CCNE-CDK2 activity decreases cell proliferation and tumor formation and is deemed a therapeutic approach in human cancers [9-12]. Therefore, better understanding of how the cell cycle transits would aid comprehension of bladder cancer progression and provide new clues for developing novel therapeutic strategies.

MNX1 (motor neuron and pancreas homeobox 1), also known as HLXB9, is a homeodomain-containing transcription factor [13]. It is located on chromosome 7q36.3 and belongs to the family of EHG homeobox genes that also includes EN1, EN2, GBX1, and GBX2 [14]. Although its normal function is unknown, it is involved in several important pathologies. First, MNX1 mutation or deletion is the primary cause of Currarino syndrome, which is a rare congenital malformation characterized by sacral anomalies, anorectal malformation and presacral mass, suggesting that deregulation of MNX1 may induce abnormal development of tissue and be associated with cell malignant transformation $[15,16]$. In addition, MNX1 is a causative oncogene in infant acute myeloid leukemias (AML) [17-20]. Interestingly, several studies have revealed that MNX1 is upregulated in some solid human cancers. Neufing et al. suggested that MNX1 is overexpressed in breast carcinoma compared to the corresponding non-malignant tissue [21]. In agreement with this, Wilkens et al. confirmed HLXB9 upregulation in surgical specimens in a subgroup of poorly differentiated hepatocellular carcinoma [22]. It was also recently reported that MNX1 is a novel oncogene upregulated to a relatively greater degree in prostate cancer [23]. Taken together, MNX1 overexpression may play an important role in tumor development. However, its biological roles and detailed molecular mechanism in human bladder cancer remain unknown.

Herein, we show that MNX1 is obviously upregulated in bladder cancer cells and is associated with poorer prognosis. MNX1 overexpression markedly promoted bladder cancer cell proliferation and tumorigenicity both in vitro and in vivo, whereas MNX1 silencing inhibited it. Furthermore, we found that MNX1 can accelerate G1-S transition in the bladder cancer cell cycle by transcriptionally upregulating CCNE1 and CCNE2 expression. Our results reveal a novel mechanism for the oncogenic role of MNX1 in bladder cancer and suggest MNX1 as a new biomarker and potential therapeutic target.

\section{Methods}

\section{Human bladder cancer cell lines}

SV-HUC-1, T24, MGH-U4, 253 J, 639 V, 5637, RT4, and 575A cells were purchased from American Type Culture Collection and cultured in DMEM with $10 \%$ foetal bovine serum (FBS, HyClone, Logan, UT, USA). All cells were incubated at $37{ }^{\circ} \mathrm{C}$ in $5 \% \mathrm{CO}_{2}$ atmosphere.

\section{Patient information and tissue specimens}

This study was conducted on 167 paraffin-embedded, archived bladder cancer samples that had been histopathologically and clinically diagnosed at the Sun Yat-sen University Cancer Center from 2005 to 2011. Additional file 1: Table S1 summarizes the clinicopathological characteristics. Ethical approval from the Institutional Research Ethics Committee and prior patient consent had been obtained for the use of the clinical specimens for research purposes. Freshly collected bladder cancer tissues were frozen and stored in liquid nitrogen until used.

\section{RNA extraction, reverse transcription and real-time PCR}

Total RNA was extracted from cultured cells using the Trizol reagent (Invitrogen, Carlsbad, CA, USA) according to the manufacturer's instructions. $2 \mu \mathrm{g}$ extracted RNA from each sample was used for cDNA synthesis with M-MLV Reverse Transcriptase (Promega, Madison, US). cDNAs were amplified and quantified by SYBR-Green in CFX96 Real Time System C1000 Cycler (Bio-Rad Laboratories, Singapore). Expression data were normalized to the housekeeping gene GAPDH and calculated as $2^{-[(\mathrm{Ct} \text { of gene })-(\mathrm{Ct} \text { of GAPDH })]}$, where $\mathrm{Ct}$ represents the threshold cycle for each transcript. Primers used in the PCR reactions are listed in the Additional file 2: Table S4.

\section{Western blotting}

Cells were harvested and equal quantities of denatured protein samples were resolved on SDS-polyacrylamide gels, and then transferred onto polyvinylidene difluoride membranes. The membrane was blocked and incubated with primary antibodies, followed by the horseradixh peroxidase-conjugated secondary antibody. Proteins were visualized using ECL reagents. An anti-MNX1 rabbit polyclonal antibody (1:500 dilution; Sigma Aldrich), an anti-CCNE1 Rabbit polyclonal antibody (1:1000 dilution;Proteintech), an anti-CCNE2 Rabbit polyclonal antibody (1:1000 dilution;Proteintech), an anti- $\alpha$-tubulin mouse monoclonal antibody (1:4000 dilution; Sigma-Aldrich), an anti-Rb rabbit polyclonal antibody (1:1000 dilution; Cell Signaling Technology), an anti-p-Rb Rabbit polyclonal antibody (1:1000 dilution; Cell Signaling Technology), were used in this study. 


\section{Immunohistochemistry (IHC)}

Immunohistochemical analysis was done to study altered protein expression in 167 human bladder cancer tissues. In brief, paraffin-embedded specimens were cut into $4-\mu \mathrm{m}$ sections and baked at $65{ }^{\circ} \mathrm{C}$ for $30 \mathrm{~min}$. The sections were deparaffinized with xylenes and rehydrated. Sections were submerged into EDTA antigenic retrieval buffer and microwaved for antigenic retrieval. The sections were treated with $3 \%$ hydrogen peroxide in methanol to quench the endogenous peroxidase activity, followed by incubation with $1 \%$ bovine serum albumin to block nonspecific binding. Rabbit anti-MNX1 (1:500 dilution; Sigma Aldrich) was incubated with the sections overnight at $4{ }^{\circ} \mathrm{C}$. For negative controls, the rabbit anti-MNX1 antibody was replaced with normal goat serum, or the rabbit anti-MNX1 antibody was blocked with a recombinant MNX1 polypeptide by coincubation at $4{ }^{\circ} \mathrm{C}$ overnight preceding the immunohistochemical staining procedure. After washing, the tissue sections were treated with biotinylated anti-rabbit secondary antibody (Zymed), followed by further incubation with streptavidin-horseradish peroxidase complex (Zymed). The tissue sections were immersed in 3-amino-9-ethyl carbazole and counterstained with 10\% Mayer's hematoxylin, dehydrated, and mounted in Crystal Mount.

Two independent pathologists blinded to the clinical outcome scored and evaluated the staining results. The scores were determined by combining the proportion of positively-stained cells and the intensity of staining. Cell proportions were scored as follows: 0 , no positive cells; $1,<10 \%$ positive cells; $2,10-35 \%$ positive cells; $3, \quad 35-75 \%$ positive cells; $4,>75 \%$ positive cells. Staining intensity was graded according to the following standard: 0, no staining; 1, weak staining (light yellow); 2, moderate staining (yellow brown); 3 , strong staining (brown). The staining index (SI) was calculated as the product of the staining intensity score and the proportion of positive cells. Using this method of assessment, we evaluated protein expression by determining the SI, with possible scores of $0,1,2,3,4,6,8,9$, and 12. Samples with a SI $\geq 6$ were defined as high expression, and samples with a $\mathrm{SI}<6$ were defined as low expression. The cutoff value was determined on the basis of a measure of heterogeneity using the log-rank test with respect to 5-year overall and relapse-free survival.

\section{Plasmids, virus constructs and retroviral infection}

The human MNX1 cDNAs were PCR-amplified and cloned into the pMSCV-puro-retro vector (Clontech). Two shRNAs against MNX1 in pLKO.1-puro vector were purchased (Transheep Bio). Transfection of these plasmids was performed using the Lipofectamine 3000 reagent (Invitrogen) according to the manufacturer's instructions. Cells $\left(2 \times 10^{5}\right)$ were seeded and infected by retrovirus generated by pMSCV-puro-cDNAs or pLKO.1-puro-shRNAs for 3 days. The stable cell lines were selected with $0.5 \mu \mathrm{g} / \mathrm{ml}$ puromycin for 7 days. The sequences of primers are provided in the Additional file 2: Table S5.

\section{3-(4,5-Dimethyl-2-thiazolyl)-2,5-diphenyl-2H-tetrazolium bromide assay}

Cells $\left(0.2 \times 10^{4}\right.$ per well $)$ were seeded in 96 -well plates. At each time point, the cells were stained with $100 \mu$ l sterile 3-(4,5-Dimethyl-2-thiazolyl)-2,5-diphenyl-2H-tetrazolium bromide (MTT) dye $(0.5 \mathrm{mg} / \mathrm{ml}$; Sigma) for $4 \mathrm{~h}$ at $37 \mathrm{C}$, followed by removal of the culture medium and addition of $150 \mu \mathrm{l}$ of dimethyl sulphoxide (Sigma). The absorbance was measured at $570 \mathrm{~nm}$, with $655 \mathrm{~nm}$ as the reference wavelength. All experiments were performed in triplicate.

\section{Colony formation}

Cells were plated on 6 well-plates $\left(0.5 \times 10^{3}\right.$ cells per plate) and cultured for 10 days. The colonies were stained with $1 \%$ crystal violet for $30 \mathrm{~s}$ after fixation with $10 \%$ formaldehyde for $5 \mathrm{~min}$.

\section{Bromodeoxyuridine (BrdU) incorporation assay}

The bladder cancer cells were grown on cover slips. BrdU was added to the cells and incubated for $2 \mathrm{~h}$. The cells were then fixed and stained with anti-BrdU antibody (Upstate, Temecula, CA, USA) according to the manufacturer's instructions. The percentage of BrdU-positive cells in five random low-power fields was counted and reported as the mean $\pm \mathrm{SD}$.

\section{Luciferase activity assay}

CCNE1 promoter from -1750 to +350 and CCNE2 promoter from -2450 to +350 were amplified by PCR, and then cloned into the pGL3 plasmid using the Sac II and Xho1 restriction enzymes. Primers for promoter amplification were: CCNE1-promoter-F: gccCCGCGG cctgttactggtgattcctaacg; R: gccCTCGAGgtgtcccctccacccca; CCNE2-promoter-F: gccAGATCTgaaaggggagactgggctg; R: gccGTCGACaaaaaaaggcacagaataaagaaat.

Luciferase assays were performed in stable cell lines with MNX1 overexpression or knockdown. Briefly, $3 \times$ $10^{4}$ stably transfected cells were cultured in triplicate in 48 -well plates for $24 \mathrm{~h}$. Then, $100 \mathrm{ng}$ luciferase reporter plasmids or the control-luciferase plasmid, plus $1 \mathrm{ng}$ pRL-TK Renilla plasmid (Promega), were transiently transfected into the indicated stable cell lines using the Lipofectamine 3000 reagent (Invitrogen), according to the manufacturer's recommendations. Luciferase and Renilla signals were measured $24 \mathrm{~h}$ after transfection, using the Dual Luciferase Reporter Assay Kit (Promega). Primers of the promoters were presented in the Additional file 2: Table S5. 


\section{Chromatin immunoprecipitation (ChIP)}

Cells $\left(4 \times 10^{6}\right)$ in a $100 \mathrm{~mm}$ culture dish were treated with $1 \%$ final concentration of formaldehyde to cross-link proteins to DNA, and the reaction was stopped by addition of glycine. The cell lysates were sonicated to shear DNA to sizes of 300-1000 bp. Equal aliquots of chromatin supernatants were incubated with $1 \mu \mathrm{g}$ of anti-MNX1, or anti-immunoglobulinG antibodies (Millipore, Billerica, MA, USA) overnight at $4{ }^{\circ} \mathrm{C}$ with rotation. After reverse cross-link of protein/DNA complexes to free DNA, PCR was performed. Specific primers for ChIP were presented in the Additional file 2: Table S6.

\section{Xenograft tumor model and tissue staining}

Male BALB/C-nu mice (5-6 weeks old, 18-20 g) were purchased from the Slac-Jingda Animal Laboratory (Hunan, China), and housed in barrier facilities on a 12-h light/dark cycle. The Institutional Animal Care and Use Committee of a

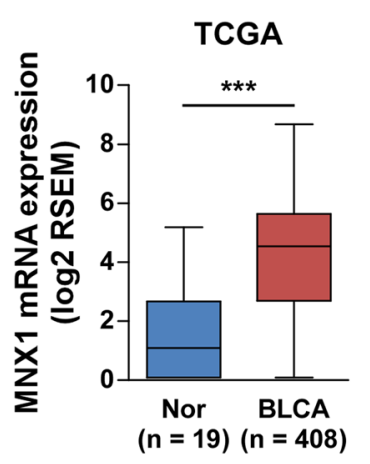

C

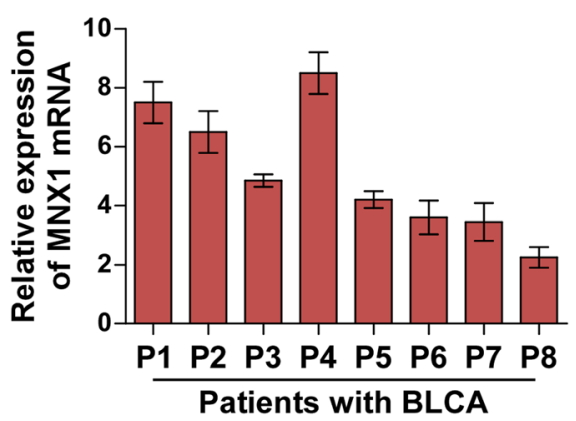

e

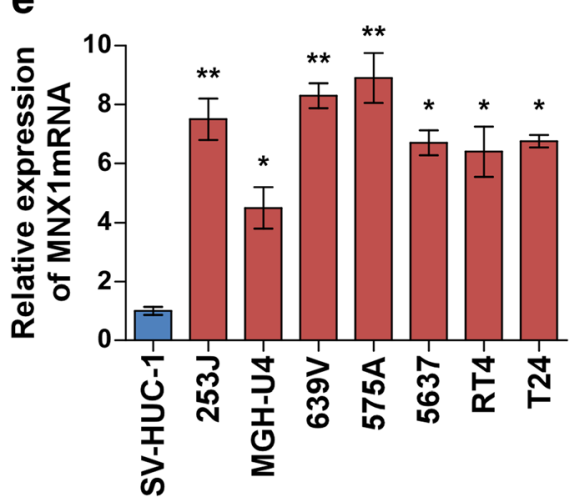

b

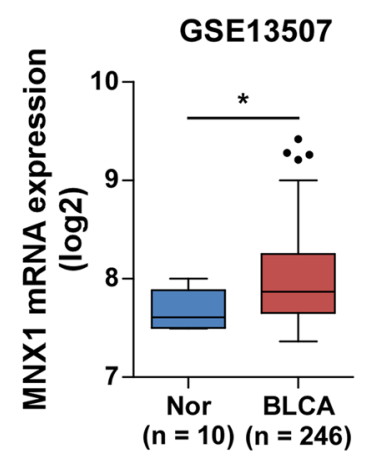

d

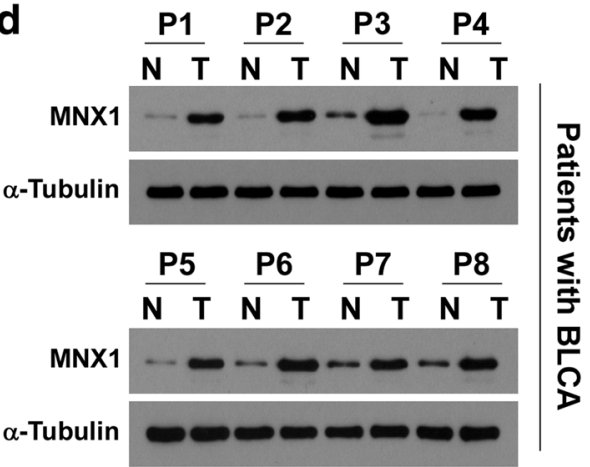

f

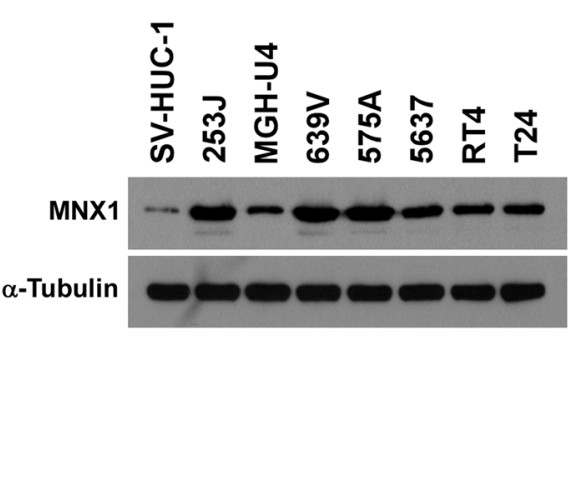

Fig. 1 MNX1 is upregulated in bladder cancer tissues and cell lines. a MNX1 mRNA expression levels in TCGA bladder cancer dataset including 19 normal bladder (Nor) and 408 bladder cancer (BLCA) samples. b MNX1 mRNA levels were significantly upregulated in bladder cancer compared to normal bladder tissues by analyzing the GEO dataset (GSE13507), including 10 normal bladder (Nor) and 246 primary bladder cancer (BLCA) tissues. c and d Real-time PCR and western blotting of MNX1 mRNA (c) and protein (d) expression levels, respectively, in bladder cancer tissues (BLCA, T) versus the adjacent

noncancerous tissues ( $\mathrm{N}$ ) from eight patients. e and $\mathbf{f}$ Real-time PCR and western blotting of MNX1 mRNA (e) and protein ( $\mathbf{f}$ ) expression levels, respectively, in seven human bladder cancer cell lines compared to the immortal SV-HUC-1 bladder cell line. Expression levels were normalized to glyceraldehyde-3phosphate dehydrogenase (GAPDH) or a-tubulin. Bars represent the mean \pm SD of three independent experiments. ${ }^{*} p<0.05 ;{ }^{* *} p<0.01 ;{ }^{* *} p<0.001$ 
Sun Yat-sen University approved all experimental procedures. The mice were randomly assigned to groups $(n=8$ per group) and their dorsal flanks were subcutaneously injected with $1 \times 10^{6}$ T24 cells. After 7 days, tumor formation kinetics were estimated by measuring tumor size at 3-day intervals. Tumor volume was calculated using the eq. $\left(\mathrm{L}^{*} \mathrm{~W}^{2}\right) / 2$. The animals were euthanized on day 42, and the tumors were excised, weighed, and paraffin-embedded. Serial $6.0-\mu \mathrm{m}$ sections were obtained and stained with anti-Ki-67 (Dako, Glostrup, Denmark) and anti-BrdU.

\section{Statistical analysis}

Statistical analyses were performed using the SPSS version 19.0 (SPSS Inc.) statistical software package. The log-rank test, $X^{2}$ test, Spearman rank correlation test, and Student $t$-test (two-tailed) were used. Multivariate statistical analysis was performed using a Cox regression model. Data are the mean \pm SD. $P<0.05$ was considered statistically significant.

\section{Results \\ MNX1 was markedly upregulated in bladder cancer}

To investigate the spectrum of MNX1 expression in bladder cancer, we first analyzed MNX1 expression in publicly available human bladder cancer datasets from The Cancer Genome Atlas (TCGA) [24] and Gene Expression Omnibus (GEO) [25]. MNX1 mRNA levels were obviously upregulated in bladder cancer samples compared with normal bladder tissues (Fig. 1a, b). We then detected MNX1 expression in bladder cancer cell lines and tissues. Western blotting and real-time PCR showed that MNX1 mRNA and protein expression, respectively, were markedly upregulated in all bladder cancer cell lines compared to primary normal urethral epithelial cells (Fig. 1c, d). Consistently, MNX1 expression was significantly higher in eight human bladder cancer tissues than in the paired adjacent

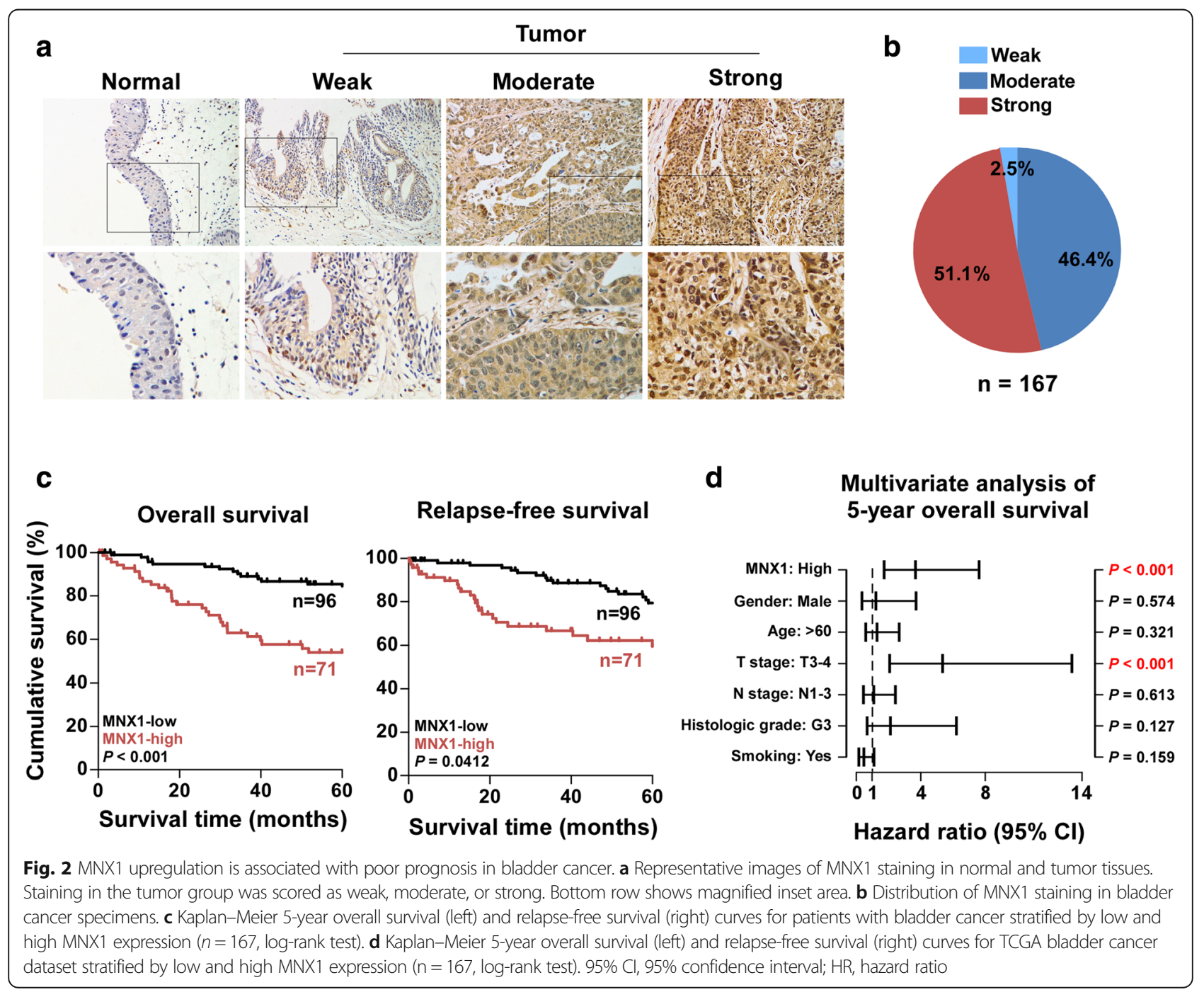


non-tumor tissues (Fig. 1e, f). These findings demonstrate that MNX1 is upregulated in bladder cancer.

To evaluate the relationship between MNX1 expression and the clinicopathological features of bladder cancer, we analyzed 167 human bladder cancer tissue samples by IHC. Similarly, IHC indicated that MNX1 was greatly overexpressed in bladder cancer samples (Fig. 2a, b). The potential correlation between the expression status of MNX1 and clinical pathologic parameters of patients with bladder cancer was further analyzed (Additional file 1: Table S2). Furthermore, Kaplan-Meier survival curves and log-rank testing showed that patients with high MNX1 expression had shorter 5-year overall survival and relapse-free survival than those with low MNX1 expression (Fig. 2c). In addition, univariate Cox regression analysis indicated that the MNX1 expression and T stage status were each recognized as independent prognostic factors for the 5-year overall survival in bladder cancer (Fig. 2d and Additional file 1: Table S3). Taken together, these results show that MNX1 is upregulated in bladder cancer cell lines and tissues, and that it might lead to poor clinical outcome.

\section{MNX1 overexpression accelerated bladder cancer cell proliferation}

To further investigate the effect of MNX1 in bladder cancer progression, we first established MNX1 stable overexpression and knockdown in T24 and 5637 cell lines (Fig. 3a). Strikingly, we found that MNX1 overexpression enhanced bladder cancer cell proliferation. The tetrazolium (MTT) assay showed that MNX1 upregulation increased the proliferation rate of bladder cancer cells; MNX1 depletion reduced it (Fig. 3b). The subsequent colony formation assay showed that MNX1 promoted bladder cancer cell colony numbers significantly, while silencing MNX1 had the opposite effects, and the colony numbers were counted and shown in histogram (Fig. 3c and d). These data suggest that MNX1 plays an important role in bladder cancer cell proliferation.

\section{Upregulating MNX1 drove G1-S transition in bladder cancer cells}

To further evaluate whether this MNX1-induced promotion of cell proliferation was due to the inhibition of cell cycle arrest, we examined the effect of MNX1 on cell cycle of bladder cancer cells. Cell lines stably overexpressing MNX1 had significantly increased proportions of cells in the $\mathrm{S}$ phase, but reduced proportions of cells in the G1 phase. In contrast, silencing MNX1 had the opposite effects (Fig. 4a). The BrdU incorporation assay showed that the percentages of BrdU-incorporating cells were significantly enhanced in MNX1-overexpressing cells and were reduced in MNX1-silenced cells (Fig. 4b). These results indicate that

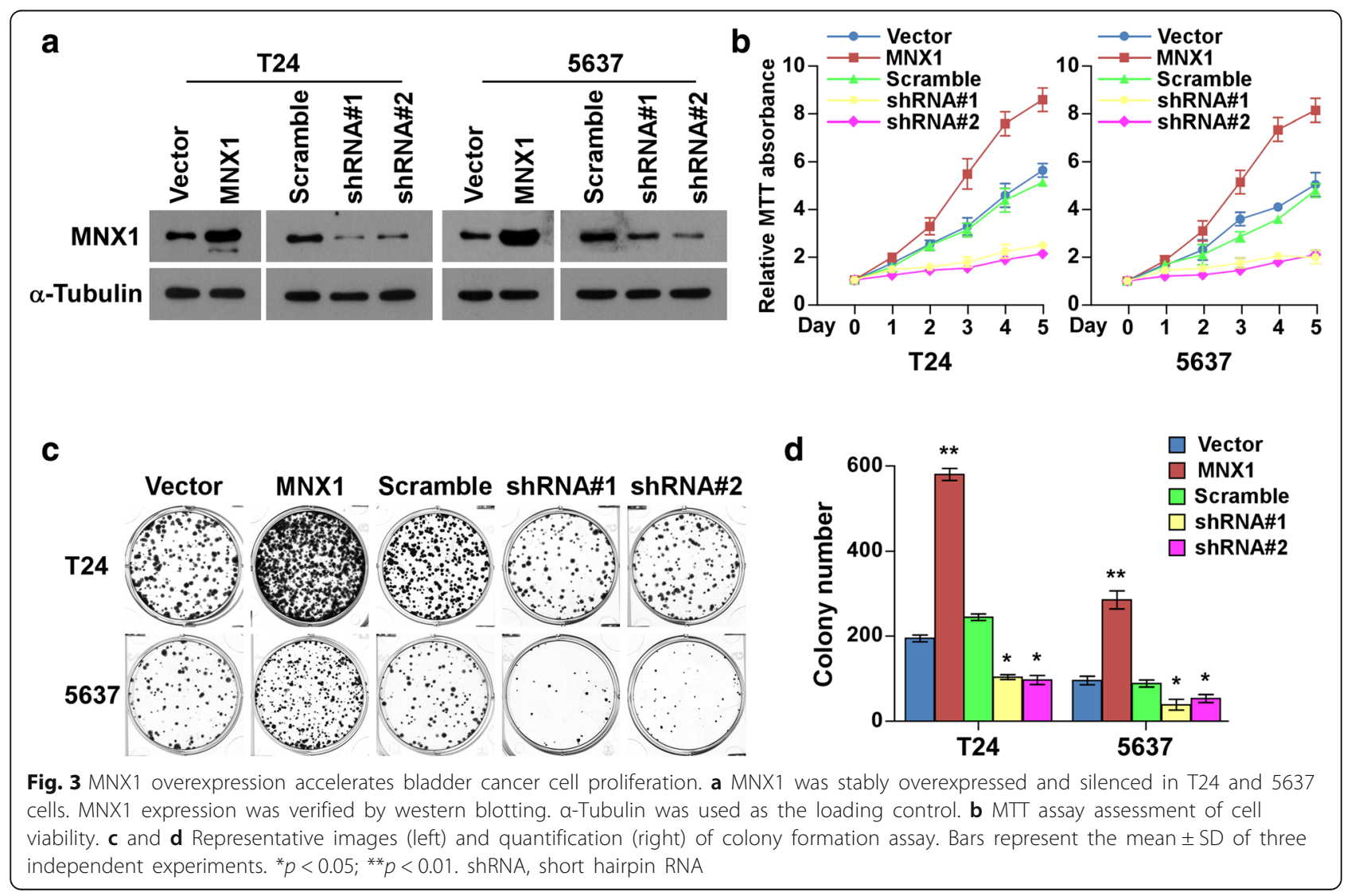




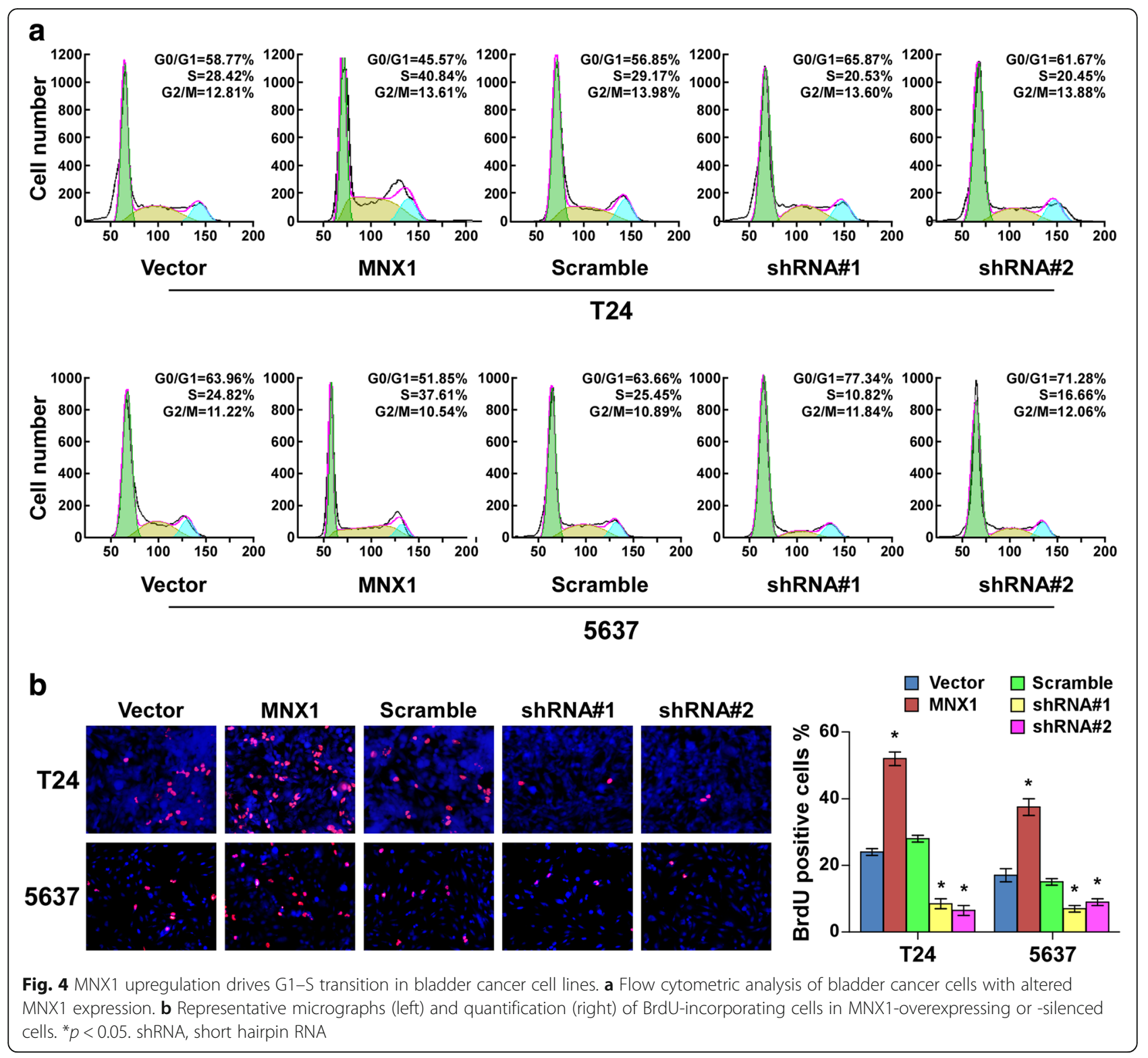

MNX1 promotes cell cycle progression of bladder cancer cells, confirming that MNX1 promotes bladder cancer cell proliferation.

\section{MNX1 promoted bladder cancer cell tumorigenicity in} vivo

To further explore the effects of MNX1 in bladder cancer tumorigenesis in vivo, we established MNX1 stable overexpression and knockdown in T24 cells (Fig. 5a). MNX1-overexpressing, MNX1 knockdown, or vector cells were subcutaneously injected into BALB/c-nu mice, and the tumor growth was measured. The MNX1-overexpressing group had prominently increased tumor growth, whereas it was obviously suppressed in the MNX1 knockdown group, both as compared with the vector group (Fig. 5b, c). Moreover, tumors from the MNX1 upregulation group exhibited high expression of the proliferation marker Ki-67 compared to the vector group. In contrast, MNX1 downregulation was associated with low Ki-67 expression (Fig. 5d). These results indicate that MNX1 promotes bladder cancer cell growth in vivo.

MNX1 directly upregulated CCNE1 and CCNE2 promoter activity in bladder cancer cells

As MNX1 is involved in the cell cycle regulation of bladder cancer cells, we examined the expression of cell cycle regulators. Real-time PCR and western blotting revealed that multiple cell cycle regulators, especially CCNE1 and CCNE2, were robustly increased in MNX1-overexpressing cells, but were reduced in 

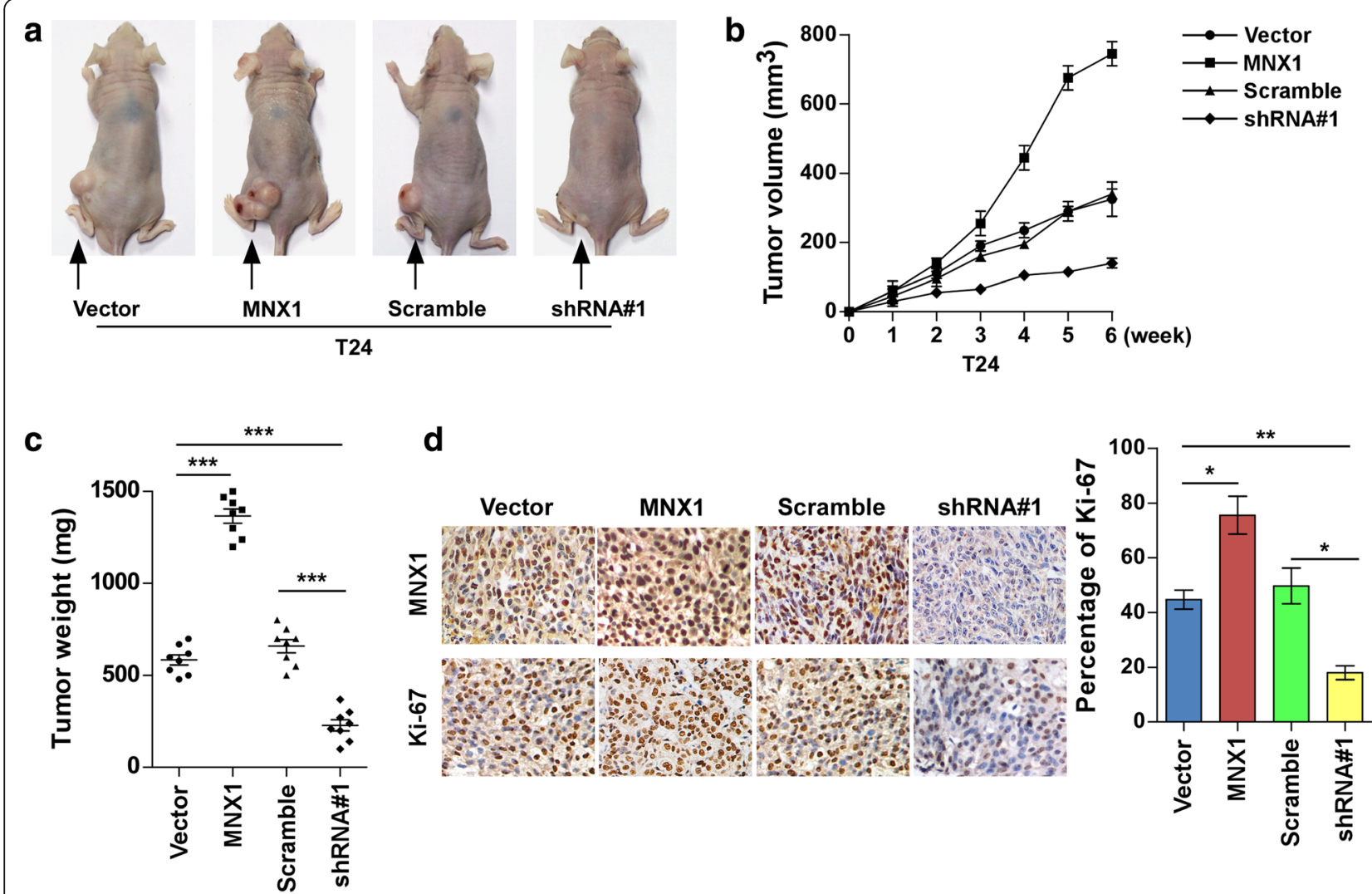

Fig. 5 MNX1 promotes bladder cancer cell tumorigenicity in vivo. a Images show one representative mouse from each group. $\mathbf{b}$ Tumor volumes were calculated every week. c The primary tumors were excised and weighed at 6 weeks after transplantation. $\mathbf{d} I \mathrm{HC}$ staining and histogram of Ki-67 in xenografts. ${ }^{*} p<0.05,{ }^{* *} p<0.001$. shRNA, short hairpin RNA

MNX1-silenced cells compared to the control cells (Fig. 6a, b). Moreover, phosphorylation of Rb, the downstream target protein of the CCNE-CDK2 complex, was induced in MNX1-overexpressing cells, but was suppressed in the MNX1-silenced cells (Fig. 6b). Consistently, using the cBioportal program (http://www.cbioportal.org), we found that most of these regulators were positively correlated with MNX1 expression (Additional file 3: Figure S1). Intriguingly, among these cyclin genes, CCNE1 and CCNE2 showed the highest correlation with MNX1 $(r=0.32$ and 0.26$)$, suggesting that MNX1 might play a role in CCNE1/2 transcription (Fig. 6c). As expected, luciferase reporter assays revealed that MNX1 overexpression activated the luciferase activity of CCNE1 and CCNE2 promoters in the bladder cancer cells, whereas MNX1 downregulation attenuated it (Fig. 6d, e). We then examined whether MNX1 upregulates CCNE1 and CCNE2 transcriptionally using chromatin immunoprecipitation (ChIP). The ChIP showed that MNX1 can bind to different regions within the CCNE1 and CCNE2 promoters (Fig. $6 \mathrm{~d}, \mathrm{e}$ ). The above data indicate that MNX1 regulates CCNE1 and CCNE2 by directly targeting their promoter elements.

\section{Discussion}

MNX1 protein has been proven to be an important regulator of many processes relevant to cancer. For example, MNX1 is involved in a recurrent translocation specifically found in infant AML, in which the MNX1 gene is frequently fused to the ETV6 gene on chromosome 1 [14]. It was also recently determined that MNX1 plays a significant role in cell proliferation in human insulinomas [26]. Moreover, MNX1 is associated with normal cells malignant transformation depending on its mutation causes congenital malformation-Currarino syndrome, suggesting that MNX1 may be a potential driver in tumorigenesis. However, the clinical importance and biological role of MNX1 in bladder cancer remain largely unknown. Herein, we found that MNX1 was robustly upregulated in bladder cancer, and established a vital role for MNX1 as a tumor-promoting factor of bladder cancer proliferation and tumorigenicity. Strikingly, IHC could detect MNX1 at all T classifications in the bladder cancer specimens and it correlated with poor outcome. Therefore, our results suggest that MNX1 may be an oncogene and might represent a novel prognostic biomarker in bladder cancer. 

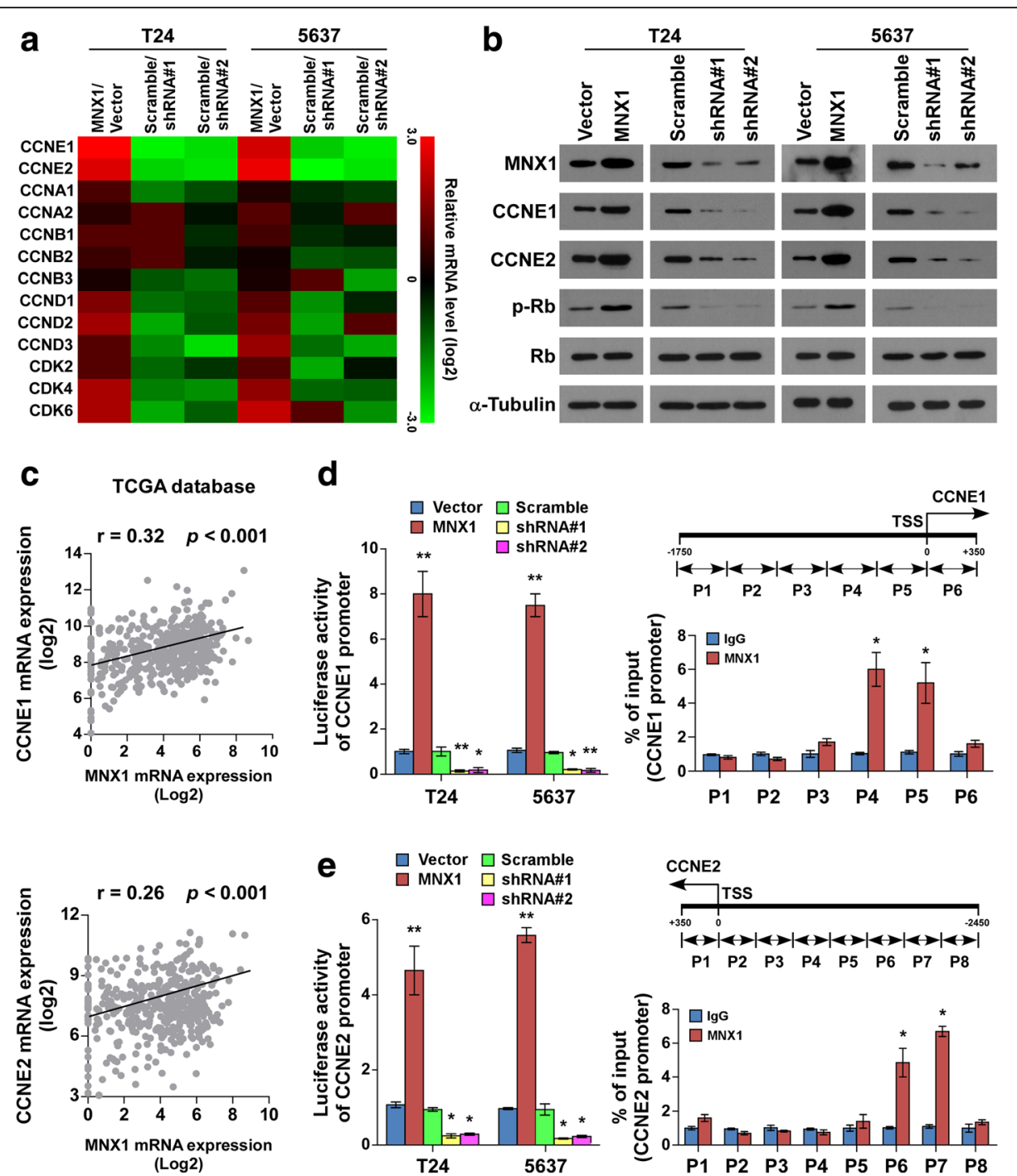

Fig. 6 MNX1 directly upregulates CCNE1 and CCNE2 promoter activity in bladder cancer cells. a Real-time PCR of cell cycle-related gene mRNA expression. Gene expression levels were normalized to GAPDH. Pseudo-colors represent the intensity scale of MNX1 versus vector or MNX1 shRNA\#1/2 versus Scramble, generated by log2 transformation. b Western blotting of CCNE1, CCNE2, phosphorylated Rb (p-Rb), and total Rb protein expression; a-tubulin was used as the loading control. c The scatter diagram of CCNE1 and CCNE2 from cBioportal program. $\mathbf{d}$ and $\mathbf{e}$ Left: Luciferase activity assays of T24 and 5637 cells showed transactivation and repression of the CCNE1 (d) and CCNE2 (e) promoters by MNX1 overexpression and MNX1 knockdown, respectively. Right: Schematic illustration of ChIP PCR fragments for the indicated nucleotide regions of the CCNE1 (d) and CCNE2 (e) promoters (top). The ChIP enrichment assay confirmed that MNX1 binds to the P4 and P5 promoter of CCNE1 (d) and the P6 and P7 promoters of CCNE2 (e); immunoglobulin G (lgG) was used as the negative control. The results from three independent experiments were evaluated. ${ }^{*} p<0.05 ;{ }^{* *} p<0.01$. shRNA, short hairpin RNA

Patients with early-stage or localized bladder cancer can be managed by surgical resection, while those with advanced bladder cancer are usually treated with radiotherapy or chemotherapy. Although efficient treatment is administered, the therapeutic outcomes remain unsatisfactory [27]. Our data reveal that ectopic expression of MNX1 promoted the formation of subcutaneous tumors in vivo, while silencing MNX1 inhibited it, which indicates MNX1 is an important factor in bladder cancer cell tumorigenicity. Our findings suggest that MNX1 is a potential therapeutic target against bladder cancer.
Generally, dysregulation of CCNE1/2 activity is present in various cancers [28-31], resulting in disrupted G1-S transition and uncontrolled cell proliferation. In the present work, MNX1 overexpression induced the expression of multiple cell cycle regulators and upregulated CCNE1 and CCNE2 by directly targeting their promoter elements, leading to G1-S transition and a high cell proliferation rate. Involvement of the CCNE-CDK2 complex is well-established in cell cycle regulation, playing an important role in tumor development [32, 33]. The E2F transcription factors strongly activate CCNE1 and CCNE2, 
and the CCNE-CDK2 complex phosphorylates and inactivates $\mathrm{Rb}$, while the phosphorylated $\mathrm{Rb}$ releases E2F transcription factors, thereby promoting cell cycle progression from G1 to S phase [34-37]. Herein, we investigated that MNX1 upregulates CCNE1 and CCNE2 expression to induce proliferation and tumorigenicity in bladder cancer by targeting their promoters. However, we have not figured out the specific sequence of CCNE1 and CCNE2 that MNX1 directly binds to. The detail mechanisms remain to be clarified in future. These observations reveal a new molecular mechanism of MNX1 in bladder cancer.

\section{Conclusions}

In summary, our study reveals that MNX1 upregulation plays an important role in bladder cancer progression and that it is a critical cell cycle promoter that upregulates CCNE1 and CCNE2 directly. However, more MNX1 mechanisms and functions in bladder cancer require further exploration. These findings will not only advance our understanding of the mechanism underlying cell cycle regulation and tumorigenicity, but also establish MNX1 as a key regulator of bladder cancer progression and a valuable prognostic marker, and may also facilitate the development of new therapeutic strategies against bladder cancer.

\section{Additional files}

\section{Additional file 1: Table S1. Clinicopathological characteristics of 167 patient samples. Table S2. Correlation between MNX1 and clinicopathological characteristics of bladder cancer patients. Table S3. Univariate and multivariate analysis of factors associated with overall survival in 167 bladder cancer patients. (DOCX $24 \mathrm{~kb}$ )}

Additional file 2: Table S4. Primers for real-time PCR analysis. Table S5. Primers for plasmid constructs. Table S6. Primers for ChIP. (DOCX 18 kb)

Additional file 3: Figure S1. Pearson score of the indicated cell cycle regulators from cbioportal. (DOCX $83 \mathrm{~kb}$ )

\section{Abbreviations}

BrdU: Bromodeoxyuridine; CCNE1/2: Cyclin E1/2; CDK: Cyclin-dependent kinase; ChIP: Chromatin immunoprecipitation; GEO: Gene Expression Omnibus; IHC: Imunohistochemistry; MNX1: Motor neuron and pancreas homeobox 1; Rb: Retinoblastoma; TCGA: The Cancer Genome Atlas

\section{Acknowledgements}

We would like to thank Fang-jian Zhou and Mu-sheng Zeng for designing and supporting this experiment.

\section{Funding}

This work was supported by the Natural Science Foundation of Guangdong Province [No. 2014A030310017, No.2017A030313686, No. S2013010015356, and No.2015A030313733].

\section{Availability of data and materials}

The datasets used and analyzed in the current study are available from the corresponding author in response to reasonable requests.

\section{Authors' contributions}

$M C, R W$ and $G L$ contributed equally to this work. MC carried out the major experiments and writing the manuscript; RW performed the statistical analysis; GL collected tissue samples. YY carried out the patients' follow-up. $\mathrm{CL}$ provided clinical tissue samples. KX performed the histological examination of the bladder tumors. LT carried out animal experiments. ZQ was responsible for the design of the study. All authors have read and approved the final version of manuscript.

Ethics approval and consent to participate

The collection and use of the bladder cancer tissue samples were reviewed and approved by the Institutional Research Ethics Committee of Sun Yat-sen University Cancer Center (China).

\section{Consent for publication}

Not applicable.

\section{Competing interests}

The authors declare that they have no competing interests.

\section{Publisher's Note}

Springer Nature remains neutral with regard to jurisdictional claims in published maps and institutional affiliations.

\section{Author details}

'Department of Urology, The Third Affiliated Hospital of Southern Medical University, Guangzhou 510630, Guangdong, China. ²Department of Urology, the First Affiliated Hospital, Sun Yat-sen University, Guangzhou 510080, Guangdong, China. ${ }^{3}$ Department of Urology, Guangzhou Red Cross Hospital, The Affiliated Hospital of Medical College of Ji-Nan University, Guangzhou 510220, Guangdong, China. ${ }^{4}$ Department of Urology, Sun Yat-sen University Cancer Center, State Key Laboratory of Oncology in South China, Collaborative Innovation Center for Cancer Medicine, Guangzhou 510060, Guangdong, China. ${ }^{5}$ Department of Urology, Cancer Center, Sun Yat-sen University, Guangzhou, Guangdong 510060, China.

Received: 26 February 2018 Accepted: 5 July 2018

Published online: 16 July 2018

References

1. Torre LA, Bray F, Siegel RL, Ferlay J, Lortet-Tieulent J, Jemal A. Global cancer statistics, 2012. CA Cancer J Clin. 2015;65(2):87-108.

2. Miyazaki J, Nishiyama H. Epidemiology of urothelial carcinoma. Int J Urol. 2017;

3. Hanahan D, Weinberg RA. Hallmarks of cancer: the next generation. Cell. 2011;144(5):646-74.

4. Frolov MV, Dyson NJ. Molecular mechanisms of E2F-dependent activation and pRB-mediated repression. J Cell Sci. 2004;117(Pt 11):2173-81.

5. Lopez-Beltran A, MacLennan GT, Montironi R. Cyclin E as molecular marker in the management of breast cancer: a review. Anal Quant Cytol Histol. 2006;28(2):111-4.

6. Hunt KK, Keyomarsi K. Cyclin E as a prognostic and predictive marker in breast cancer. Semin Cancer Biol. 2005;15(4):319-26.

7. Muller-Tidow C, Metzger R, Kugler K, Diederichs S, Idos G, Thomas M, DockhornDworniczak B, Schneider PM, Koeffler HP, Berdel WE, et al. Cyclin E is the only cyclin-dependent kinase 2-associated cyclin that predicts metastasis and survival in early stage non-small cell lung cancer. Cancer Res. 2001;61(2):647-53.

8. Akli S, Zhang XQ, Bondaruk J, Tucker SL, Czerniak PB, Benedict WF, Keyomarsi K. Low molecular weight cyclin $\mathrm{E}$ is associated with p27-resistant, high-grade, high-stage and invasive bladder cancer. Cell Cycle. 2012;11(7):1468-76.

9. Fu YP, Kohaar I, Moore LE, Lenz P, Figueroa JD, Tang W, Porter-Gill P, Chatterjee N, Scott-Johnson A, Garcia-Closas M, et al. The 19q12 bladder cancer GWAS signal: association with cyclin E function and aggressive disease. Cancer Res. 2014;74(20):5808-18.

10. Han B, Luan L, Xu Z, Wu B. Expression and biological roles of SATB1 in human bladder cancer. Tumour Biol. 2013;34(5):2943-9.

11. Jiang Y, Han Y, Sun C, Han C, Han N, Zhi W, Qiao Q. Rab23 is overexpressed in human bladder cancer and promotes cancer cell proliferation and invasion. Tumour Biol. 2016;37(6):8131-8.

12. Matsushita R, Seki N, Chiyomaru T, Inoguchi S, Ishihara T, Goto Y, Nishikawa R, Mataki H, Tatarano S, Itesako T, et al. Tumour-suppressive microRNA-144$5 p$ directly targets CCNE1/2 as potential prognostic markers in bladder cancer. Br J Cancer. 2015;113(2):282-9.

13. Holland PWH. Beyond the Hox: how widespread is homeobox gene clustering? J Anat. 2001;199(1):13-23.

14. Nagel S, Kaufmann M, Scherr M, Drexler HG, MacLeod RA. Activation of HLXB9 by juxtaposition with MYB via formation of $t(6 ; 7)(q 23 ; q 36)$ in an AML-M4 cell line (GDM-1). Genes Chromosomes Cancer. 2005;42(2):170-8. 
15. Belloni E, Martucciello G, Verderio D, Ponti E, Seri M, Jasonni V, Torre M, Ferrari M, Tsui LC, Scherer SW. Involvement of the HLXB9 homeobox gene in Currarino syndrome. Am J Hum Genet. 2000;66(1):312-9.

16. Merello E, De Marco P, Ravegnani M, Riccipetitoni G, Cama A, Capra V. Novel MNX1 mutations and clinical analysis of familial and sporadic Currarino cases. Eur J Med Genet. 2013;56(12):648-54.

17. Beverloo HB, Panagopoulos I, Isaksson M, van Wering $E$, van Drunen $E$, de Klein A, Johansson B, Slater R. Fusion of the homeobox gene HLXB9 and the ETV6 gene in infant acute myeloid leukemias with the t(7;12)(q36;p13). Cancer Res. 2001;61(14):5374-7.

18. Taketani T, Taki T, Sako M, Ishii T, Yamaguchi S, Hayashi Y. MNX1-ETV6 fusion gene in an acute megakaryoblastic leukemia and expression of the MNX1 gene in leukemia and normal B cell lines. Cancer Genet Cytogenet. 2008;186(2):115-9.

19. Park J, Kim M, Lim J, Kim Y, Han K, Lee J, Chung NG, Cho B, Kim HK. Threeway complex translocations in infant acute myeloid leukemia with $\mathrm{t}(7$; 12)(q36;p13): the incidence and correlation of a HLXB9 overexpression. Cancer Genet Cytogenet. 2009;191(2):102-5.

20. Ferguson S, Gautrey HE, Strathdee G. The dual role of HLXB9 in leukemia. Pediatr Blood Cancer. 2011;56(3):349-52.

21. Neufing PJ, Kalionis B, Horsfall DJ, Ricciardelli C, Stahl J, Vivekanandan S, Raymond W, Tilley WD. Expression and localization of homeodomain proteins DLX4/HB9 in normal and malignant human breast tissues. Anticancer Res. 2003;23(2b):1479-88.

22. Wilkens $L$, Jaggi $R$, Hammer $C$, Inderbitzin D, Giger $O$, von Neuhoff $N$. The homeobox gene HLXB9 is upregulated in a morphological subset of poorly differentiated hepatocellular carcinoma. Virchows Arch. 2011;458(6):697-708.

23. Zhang L, Wang J, Wang Y, Zhang Y, Castro P, Shao L, Sreekumar A, Putluri N, Guha N, Deepak S, et al. MNX1 is Oncogenically upregulated in AfricanAmerican prostate Cancer. Cancer Res. 2016;76(21):6290-8.

24. Cancer Genome Atlas Research N, Weinstein JN, Collisson EA, Mills GB, Shaw KR, Ozenberger BA, Ellrott K, Shmulevich I, Sander C, Stuart JM. The Cancer genome atlas pan-Cancer analysis project. Nat Genet. 2013;45(10):1113-20

25. Clough E, Barrett T. The gene expression omnibus database. Methods Mol Biol. 2016;1418:93-110

26. Desai SS, Modali SD, Parekh VI, Kebebew E, Agarwal SK. GSK-3beta protein phosphorylates and stabilizes HLXB9 protein in insulinoma cells to form a targetable mechanism of controlling insulinoma cell proliferation. J Biol Chem. 2014;289(9):5386-98.

27. Guillaume L, Guy L. Epidemiology of and risk factors for bladder cancer and for urothelial tumors. Rev Prat. 2014;64(10):1372-4. 78-80

28. Natrajan R, Mackay A, Wilkerson PM, Lambros MB, Wetterskog D, Arnedos M, Shiu KK, Geyer FC, Langerod A, Kreike B, et al. Functional characterization of the 19q12 amplicon in grade III breast cancers. Breast Cancer Res. 2012;14(2):R53.

29. Au-Yeung G, Lang F, Azar WJ, Mitchell C, Jarman KE, Lackovic K, Aziz D, Cullinane C, Pearson RB, Mileshkin L, et al. Selective targeting of cyclin E1amplified high-grade serous ovarian Cancer by cyclin-dependent kinase 2 and AKT inhibition. Clin Cancer Res. 2017;23(7):1862-74.

30. Erdmann K, Kaulke K, Rieger C, Salomo K, Wirth MP, Fuessel S. MiR-26a and miR138 block the G1/S transition by targeting the cell cycle regulating network in prostate cancer cells. J Cancer Res Clin Oncol. 2016;142(11):2249-61.

31. da Silva GN, de Camargo EA, Salvadori DM. Toxicogenomic activity of gemcitabine in two TP53-mutated bladder cancer cell lines: special focus on cell cycle-related genes. Mol Biol Rep. 2012;39(12):10373-82.

32. Tashiro E, Tsuchiya A, Imoto M. Functions of cyclin D1 as an oncogene and regulation of cyclin D1 expression. Cancer Sci. 2007;98(5):629-35.

33. Deane NG, Parker MA, Aramandla R, Diehl L, Lee WJ, Washington MK, Nanney LB, Shyr Y, Beauchamp RD. Hepatocellular carcinoma results from chronic cyclin D1 overexpression in transgenic mice. Cancer Res. 2001;61(14):5389-95.

34. Burkhart DL, Sage J. Cellular mechanisms of tumour suppression by the retinoblastoma gene. Nat Rev Cancer. 2008;8(9):671-82.

35. Goodrich DW. The retinoblastoma tumor-suppressor gene, the exception that proves the rule. Oncogene. 2006;25(38):5233-43.

36. Taylor-Harding B, Aspuria PJ, Agadjanian H, Cheon DJ, Mizuno T, Greenberg D, Allen JR, Spurka L, Funari V, Spiteri E, et al. Cyclin E1 and RTKVRAS signaling drive CDK inhibitor resistance via activation of E2F and ETS. Oncotarget. 2015;6(2):696-714.

37. Asghar U, Witkiewicz AK, Turner NC, Knudsen ES. The history and future of targeting cyclin-dependent kinases in cancer therapy. Nat Rev Drug Discov. 2015;14(2):130-46.

\section{Ready to submit your research? Choose BMC and benefit from:}

- fast, convenient online submission

- thorough peer review by experienced researchers in your field

- rapid publication on acceptance

- support for research data, including large and complex data types

- gold Open Access which fosters wider collaboration and increased citations

- maximum visibility for your research: over $100 \mathrm{M}$ website views per year

At BMC, research is always in progress.

Learn more biomedcentral.com/submissions 